\title{
Checkpoint Inhibitors in Relapsed/Refractory Classical Hodgkin Lymphoma
}

\author{
Kidist Tarekegn a, d, e, Ana Colon Ramos ${ }^{\text {a, d }}$, Balraj Singh ${ }^{\mathrm{b}}$, \\ Harry G. Sequeira Gross ${ }^{\mathrm{a}}$, Sachin Gupta ${ }^{\mathrm{c}}$
}

\begin{abstract}
Even though classical Hodgkin lymphoma is highly curable, the outcome of patients with a refractory or relapsed disease has been disappointing. Multiple lines of therapy are available for patients after their first failure, and most respond to subsequent therapies. However, there is a sizable proportion that remains relapsing/recurrent even after several lines of therapy. The overall prognosis of patients with relapsing and recurrent classical Hodgkin lymphoma (rreHL) has been very disappointing until recently. Immune checkpoint inhibitors such as the anti-programmed death 1 (PD-1) receptor antibodies have recently been approved to treat relapsed and refractory $\mathrm{cHL}$ and have significantly improved the outcome of patients with rrcHL. The approved immune checkpoint inhibitors for relapsed and refractory cHL are nivolumab and pembrolizumab. In the Checkmate 205 study nivolumab demonstrated an objective response rate of $69 \%$ with an acceptable safety profile. Similarly, pembrolizumab demonstrated an overall response rate (ORR) of $69 \%$ with a complete remission rate (CRR) of $22.4 \%$ in the KEYNOTE-087 study in heavily pretreated patients with rrcHL.
\end{abstract}

Keywords: Checkpoint inhibitors; Nivolumab; Pembrolizumab; Relapsed/refractory classical Hodgkin lymphoma

\section{Introduction}

The development in the treatment of Hodgkin lymphoma has been an amazing multidisciplinary effort throughout history.

Manuscript submitted May 9, 2021, accepted June 3, 2021

Published online July 10, 2021

aDepartment of Internal Medicine, St. Barnabas Hospital, Bronx, NY 10457, USA

${ }^{b}$ Department of Hematology/Oncology, Saint Joseph's University Medical Center, Paterson, NJ 07503, USA

'Hospital Medicine, Tower Health Reading Hospital, West Reading, PA 19611, USA

dThese authors contributed equally to this work.

${ }^{\mathrm{e} C o r r e s p o n d i n g ~ A u t h o r: ~ K i d i s t ~ T a r e k e g n, ~ D e p a r t m e n t ~ o f ~ I n t e r n a l ~ M e d i c i n e, ~}$

St. Barnabas Hospital, Bronx, NY 10457, USA.

Email: kwtarekegn@gmail.com

doi: https://doi.org/10.14740/wjon1388
The present modalities of therapy have converted a fatal disease to one that is curable in most patients. The current guidelines for the treatment of Hodgkin lymphoma rely mostly on the stage of the disease at first presentation and the prognostic factors, which may be favorable or unfavorable. It is important to make this distinction because the therapy tends to be more aggressive in the unfavorable group. The core of classical Hodgkin lymphoma (cHL) management is based on the ABVD therapy combination (doxorubicin, bleomycin, vinblastine, dacarbazine) followed by the recommended form of radiation in the early and advanced stages of the disease [1]. Other therapies available for advanced stages such as escalated BEACOPP (bleomycin, etoposide, doxorubicin, cyclophosphamide, vincristine, procarbazine and prednisone) and brentuximab vedotin $(B V)+A V D$, saved for more aggressive presentation, have a longer progression-free survival without any impact in the overall survival. Stanford V (doxorubicin, vinblastine, mechlorethamine, vincristine, bleomycin, etoposide and prednisone) is another therapeutic option that incorporates radiation therapy for all patients. However, there is no advantage compared to ABVD [2-4].

Even though cHL is highly curable, around $25 \%$ of the patients with cHL have a refractory or relapsed disease after the first-line therapy [5]. High-dose chemotherapy (using a different chemotherapeutic agent) and autologous stem cell transplantation (SCT) is considered the treatment of choice in a subset of patients. However, in these patients, conventionaldose chemotherapy regimens induce low remission rates, resulting in long-term remissions in approximately $40-50 \%$ of relapsed patients and in up to $25-30 \%$ of those with primary refractory disease. Lately, cancer immunotherapy has shown promising results in high-risk relapsed/refractory $\mathrm{cHL}$ ( $\mathrm{rrCHL}$ ) patients using strategies to overcome the evasion of immune surveillance that can be classified as passive (administration of agents such as monoclonal antibodies, lymphocytes or cytokines that potentiate existing anti-tumor response) or active immunotherapy (the stimulation of self-immune system to attack tumor cells via vaccination, non-specific immunomodulation, or targeting specific antigen receptors). In this review, we will present the scientific rationale for the currently available immunotherapies in rrcHL.

\section{Checkpoint Inhibitors Therapy}

The overall prognosis of patients with $\mathrm{cHL}$ has improved over 
the decades with most patients responding well to the standard means of therapy [6]. However, there is a subgroup of patients who will have relapsed and refractory disease with poor outcomes. The goal for the treatment in the first relapsed/refractory episode in cHL is to achieve long-term disease control, which may be accomplished in most cases through the application of autologous SCT. The pre-transplant positron emission tomography (PET) negativity is one of the most crucial predictors of the outcome after autologous SCT in patients with relapsed or refractory cHL [1]. A state of complete remission before autologous SCT may be achieved using intensive combination or targeted chemotherapy.

Immune checkpoint inhibitors such as the anti-programmed death 1 (PD-1) receptor antibodies have recently been approved for the treatment of relapsed and refractory $\mathrm{cHL}$ and have shown to be effective [7]. The malignant Reed Sternberg cells in cHL overexpress programmed death-ligand 1 (PD-L1) and PD-L2, which confer them with several mechanisms to escape immune clearance [8]. One of the hallmarks of this escape is that the tumor cells are capable of immune suppressing the highly inflamed tumor microenvironment composed of T cells, B cells, macrophages, natural killer (NK) cells and neutrophils. Targeting PD-1 will restore immune function in the tumor microenvironment. This is the rationale for the use of these types of drugs in cHL.

The approved immune checkpoint inhibitors for relapsed and refractory $\mathrm{cHL}$ are nivolumab and pembrolizumab with demonstrated high response rates in several retrospective studies. Other immune checkpoint inhibitors including ipilimum$\mathrm{ab}$, sintilimab and tislelizumab have also been tried $[9,10]$.

\section{Nivolumab}

Nivolumab is a fully human immunoglobulin G4 (IgG4) monoclonal antibody selectively targeting the $\mathrm{PD}-1$ receptor to block the ligands PD-L1 and PD-L2 from binding. It has been previously used in other solid tumors. Nivolumab was first evaluated in a phase I trial which enrolled 23 patients who received extensive prior treatment regimens including $\mathrm{BV}$ and autologous SCT. Treatment with nivolumab had an overall response rate (ORR) of $87 \%$ and progression-free survival of $86 \%$ in 24 months follow-up. Nivolumab exhibited an acceptable safety profile with most adverse events being grade one and two [11]. Subsequently, the phase II study which enrolled 243 patients (Checkmate 205) was developed to further assess the clinical activity and safety of nivolumab. Checkmate 205 is a multi-center phase II study that enrolled 243 patients with rrcHL, who have failed after autologous SCT and treatment with BV, grouped into three cohorts according to their prior therapies. Most of the participants were heavily treated before enrollment, with the median number of prior lines of therapy being four. The objective response rate as assessed by an independent radiologic review committee (IRRC) was 69\%. It was very promising to note more than two-thirds of patients who failed BV treatment responded to nivolumab. The study also showed that nivolumab has an acceptable safety profile with most reported adverse event (AE) being grade one to two.
Subjectively patients overall reported improved quality of life [12]. Moreover, extended follow-up of Checkmate 205 study concluded that the response to nivolumab was frequent and durable. The ORR in the cohorts divided according to pretreatment regimen was $69 \%$ [13]. The promising results of these trials led to the US Food and Drug Administration (FDA) approval of nivolumab for patients with rreHL.

\section{Pembrolizumab}

Pembrolizumab is another humanized, high-affinity, IgG4 monoclonal antibody directed against PD-1. Pembrolizumab was evaluated in the phase IB study, KEYNOTE-013 (ClinicalTrials.gov, NCT01953692), designed to evaluate its safety and anti-tumor activity. Based on the knowledge of the role of PD-1 signaling pathway in the pathogenesis of Hodgkin lymphoma the study had an independent cohort of patients with Hodgkin lymphoma. The cohort had 31 patients. The participants had received multiple prior treatments with $55 \%$ receiving greater than or equal to five lines of therapy and all had received BV. Pembrolizumab had a high ORR of $65 \%$ at 12 weeks and has demonstrated a favorable safety profile. The median survival at the time of the study cutoff was 17.6 months. Although the $16 \%$ complete remission rate (CRR) in this study was low, the durability of the partial response (PR) (48 \%) achieved in this study was very promising. This shows in line with other studies, that achievement of complete remission with checkpoint blockade might not be necessary to derive significant clinical outcome. The progression-free survival and overall survival rates at 24 weeks were $69 \%$ and $100 \%$, respectively. Progression-free survival at 52 weeks was $46 \%$ [14]. Subsequently, a multicenter, single-arm phase II study (KEYNOTE-087) enrolled 210 patients who either had autologous SCT or are ineligible to evaluate the clinical activity of pembrolizumab in patients with rrcHL. The study grouped subjects into three cohorts based on disease progression and prior line of therapy. Similar to the studies with nivolumab, the study subjects had multiple prior lines of therapy with the median number of prior lines of therapy being four. In this study, the ORR was $69 \%$ with a CRR of $22.4 \%$; and interestingly, ORR did not vary based on the number of prior lines of therapy. Moreover, pembrolizumab has demonstrated an acceptable safety profile. Compared to nivolumab, pembrolizumab has shown to have better CRR [15]. Like nivolumab, the promising results of these trials led to the US FDA approval of pembrolizumab for patients with rrcHL.

\section{Combination Therapy}

The immune checkpoint inhibitors (nivolumab and pembrolizumab) have significantly improved the prognosis of relapsed and refractory cHL. While the improvement in overall response achieved by PD-1 blockade had been promising, there are a sizable proportion of patients who would not have a durable response [16]. Given this, combination therapy has been pursued. Combination therapies involving BV (a CD30-direct- 
ed antibody-drug conjugate) and nivolumab and ipilimumab; nivolumab and bendamustine; nivolumab with radiation therapy are being developed [17-20].

\section{Conclusions}

Over the last few years, checkpoint inhibitors have significantly changed the prognosis of patients with rrcHL. The checkpoint inhibitors, nivolumab and pembrolizumab, have demonstrated outstanding results in heavily pretreated (including BV and autologous SCT) patients with rrcHL in the Checkmate 205 and KEYNOTE-087 studies. Combination therapies involving checkpoint inhibitors are being pursued to fill the gap that remains in the management of rrcHL after checkpoint inhibitors.

\section{Acknowledgments}

None to declare.

\section{Financial Disclosure}

None to declare.

\section{Conflict of Interest}

None to declare.

\section{Author Contributions}

All authors contributed to conception, design, manuscript writing and final approval of the manuscript. All authors are accountable for all aspects of the work.

\section{Data Availability}

The authors declare that data supporting the findings of this study are available within the article.

\section{References}

1. Radford J, Illidge T, Counsell N, Hancock B, Pettengell R, Johnson P, Wimperis J, et al. Results of a trial of PETdirected therapy for early-stage Hodgkin's lymphoma. N Engl J Med. 2015;372(17):1598-1607.

2. Johnson P, Federico M, Kirkwood A, Fossa A, Berkahn L, Carella A, d'Amore F, et al. Adapted treatment guided by interim PET-CT Scan in advanced Hodgkin's lymphoma. N Engl J Med. 2016;374(25):2419-2429.

3. Viviani S, Zinzani PL, Rambaldi A, Brusamolino E, Levis A, Bonfante V, Vitolo $U$, et al. ABVD versus BEA-
COPP for Hodgkin's lymphoma when high-dose salvage is planned. N Engl J Med. 2011;365(3):203-212.

4. Chisesi T, Bellei M, Luminari S, Montanini A, Marcheselli L, Levis A, Gobbi P, et al. Long-term follow-up analysis of HD9601 trial comparing ABVD versus Stanford $\mathrm{V}$ versus $\mathrm{MOPP} / \mathrm{EBV} / \mathrm{CAD}$ in patients with newly diagnosed advanced-stage Hodgkin's lymphoma: a study from the Intergruppo Italiano Linfomi. J Clin Oncol. 2011;29(32):4227-4233.

5. Evens AM, Hutchings M, Diehl V. Treatment of Hodgkin lymphoma: the past, present, and future. Nat Clin Pract Oncol. 2008;5(9):543-556.

6. Hanahan D, Weinberg RA. Hallmarks of cancer: the next generation. Cell. 2011;144(5):646-674.

7. Shindiapina $\mathrm{P}$, Alinari L. Pembrolizumab and its role in relapsed/refractory classical Hodgkin's lymphoma: evidence to date and clinical utility. Ther Adv Hematol. 2018;9(4):89-105.

8. Roemer MG, Advani RH, Ligon AH, Natkunam Y, Redd RA, Homer H, Connelly CF, et al. PD-L1 and PD-L2 genetic alterations define classical hodgkin lymphoma and predict outcome. J Clin Oncol. 2016;34(23):2690-2697.

9. Shi Y, Su H, Song Y, Jiang W, Sun X, Qian W, Zhang $\mathrm{W}$, et al. Safety and activity of sintilimab in patients with relapsed or refractory classical Hodgkin lymphoma (ORIENT-1): a multicentre, single-arm, phase 2 trial. Lancet Haematol. 2019;6(1):e12-e19.

10. Song Y, Gao Q, Zhang H, Fan L, Zhou J, Zou D, Li W, et al. Treatment of relapsed or refractory classical Hodgkin lymphoma with the anti-PD-1, tislelizumab: results of a phase 2, single-arm, multicenter study. Leukemia. 2020;34(2):533-542.

11. Ansell SM, Lesokhin AM, Borrello I, Halwani A, Scott EC, Gutierrez M, Schuster SJ, et al. PD-1 blockade with nivolumab in relapsed or refractory Hodgkin's lymphoma. N Engl J Med. 2015;372(4):311-319.

12. Younes A, Santoro A, Shipp M, Zinzani PL, Timmerman JM, Ansell S, Armand P, et al. Nivolumab for classical Hodgkin's lymphoma after failure of both autologous stem-cell transplantation and brentuximab vedotin: a multicentre, multicohort, single-arm phase 2 trial. Lancet Oncol. 2016;17(9):1283-1294.

13. Armand P, Engert A, Younes A, Fanale M, Santoro A, Zinzani PL, Timmerman JM, et al. Nivolumab for relapsed/ refractory classic hodgkin lymphoma after failure of autologous hematopoietic cell transplantation: extended follow-up of the multicohort single-arm phase II CheckMate 205 trial. J Clin Oncol. 2018;36(14):1428-1439.

14. Armand P, Shipp MA, Ribrag V, Michot JM, Zinzani PL, Kuruvilla J, Snyder ES, et al. Programmed Death-1 blockade with pembrolizumab in patients with classical Hodgkin lymphoma after brentuximab vedotin failure. J Clin Oncol. 2016;34(31):3733-3739.

15. Chen R, Zinzani PL, Fanale MA, Armand P, Johnson NA, Brice P, Radford J, et al. Phase II study of the efficacy and safety of pembrolizumab for relapsed/refractory classic Hodgkin lymphoma. J Clin Oncol. 2017;35(19):21252132.

16. Ansell SM. Immunotherapy in Hodgkin Lymphoma: The 
Road Ahead. Trends Immunol. 2019;40(5):380-386.

17. Herrera AF, Moskowitz AJ, Bartlett NL, Vose JM, Ramchandren R, Feldman TA, LaCasce AS, et al. Interim results of brentuximab vedotin in combination with nivolumab in patients with relapsed or refractory Hodgkin lymphoma. Blood. 2018;131(11):1183-1194.

18. Hallam S. Dual Immunotherapy Adds Value in Relapsed/Refractory Hodgkin Lymphoma. Hemasphere. 2018;2(4):e137.
19. Formenti SC, Demaria S. Combining radiotherapy and cancer immunotherapy: a paradigm shift. J Natl Cancer Inst. 2013;105(4):256-265.

20. Lepik KV, Mikhailova NB, Kondakova EV, Zalyalov YR, Fedorova LV, Tsvetkova LA, Kotselyabina PV, et al. A study of safety and efficacy of nivolumab and bendamustine (NB) in patients with relapsed/refractory hodgkin lymphoma after nivolumab monotherapy failure. Hemasphere. 2020;4(3):e401. 